\title{
Evidence on scaling in health and social care: protocol for a living umbrella review
}

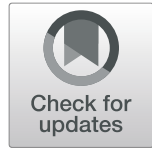

France Légaré ${ }^{1,2,3,4^{*}}$ (D), Karine V. Plourde ${ }^{1,2}$, Ali Ben Charif ${ }^{1,2}$, Amédé Gogovor ${ }^{1,2,3}$, Francesca Katherine Brundisini ${ }^{1,2,3}$, Robert K. D. McLean ${ }^{5,6}$, Andrew Milat ${ }^{7,8}$, Nathalie Rheault ${ }^{1,3}$, Luke Wolfenden ${ }^{9,10,11}$ and Hervé Tchala Vignon Zomahoun ${ }^{1,3}$

\begin{abstract}
Background: There is a growing interest in scaling effective health innovations to promote equitable access to high-quality health services worldwide. However, multiple challenges persist in scaling innovations. In this study, we aim to summarize the scaling evidence in the health and social care literature and identify current knowledge gaps.

Methods: We will conduct a living umbrella review according to the Joanna Briggs Institute Reviewers' Manual. We will consider all knowledge syntheses addressing scaling in health or social care (e.g., any setting, any clinical area) and conducted in a systematic way. We will search the following electronic databases: MEDLINE (Ovid), Embase, PsychINFO (Ovid), CINAHL (EBSCO), Web of Science, The Cochrane Library, Sociological Abstract (Proquest), Academic Search Premier (EBSCO), and Proquest Dissertations \& Theses Global, from inception. Furthermore, we will conduct searches of the grey literature. No restriction regarding date or language will be applied. Each phase of the review will be processed by two independent reviewers. We will develop a data extraction form on Covidence. We will assess the methodological quality of the included reviews using AMSTAR2 and the risk of bias using ROBIS. Results will be presented in tabular form and accompanied by a narrative synthesis covering the traditional themes of scaling science that emerge from the analysis, such as coverage, range, and sustainability, as well as themes less covered in the literature, including reporting guidance, models, tools, barriers, and/or facilitators to scaling innovations, evidence regarding application in high-income or low-income countries, and end-user engagement. We will disseminate the findings via publications and through relevant networks.
\end{abstract}

Discussion: The findings of the umbrella review will facilitate access to scaling evidence in the literature and help strengthen the science of scaling for researchers, policy makers, and program managers. Finally, this work will highlight important knowledge gaps and help prioritize future research questions.

Systematic review registration: This protocol was registered with the International Prospective Register of Systematic Reviews (PROSPERO) on November 11, 2020 (registration number: CRD42020183774).

Keywords: Scaling, Spread, Scalability, Implementation science, Scaling science, Knowledge translation, Health and social care, Living review, Umbrella review

\footnotetext{
* Correspondence: France.Legare@fmed.ulaval.ca

'VITAM - Centre de recherche en santé durable, Université Laval, Pavillon Landry-Poulin - 2525, Chemin de la Canardière, Quebec, QC, Canada

${ }^{2}$ Tier 1 Canada Research Chair in Shared Decision Making and Knowledge

Translation, Université Laval, Quebec, QC, Canada

Full list of author information is available at the end of the article
}

\section{$\triangle B M C$}

(c) The Author(s). 2021 Open Access This article is licensed under a Creative Commons Attribution 4.0 International License, which permits use, sharing, adaptation, distribution and reproduction in any medium or format, as long as you give appropriate credit to the original author(s) and the source, provide a link to the Creative Commons licence, and indicate if changes were made. The images or other third party material in this article are included in the article's Creative Commons licence, unless indicated otherwise in a credit line to the material. If material is not included in the article's Creative Commons licence and your intended use is not permitted by statutory regulation or exceeds the permitted use, you will need to obtain permission directly from the copyright holder. To view a copy of this licence, visit http://creativecommons.org/licenses/by/4.0/. The Creative Commons Public Domain Dedication waiver (http://creativecommons.org/publicdomain/zero/1.0/) applies to the data made available in this article, unless otherwise stated in a credit line to the data. 


\section{Background}

There is great interest in implementing health care innovations at a larger scale to achieve better health of populations and to reduce per capita cost of health care. Spread (replicating an innovation) and scale (building infrastructure to support full scale implementation) [1] are both used in the fields of knowledge translation (KT) and implementation science to refer to increasing the reach and adoption of innovations. The concepts of spread and scale broadly correspond with what others describe respectively as vertical and horizontal approaches. Vertical scaling up consists of using policy, regulation, or financial tools to expand an innovation simultaneously across a whole system (e.g., introduction of mandatory seatbelt legislation), while horizontal scaling up is the phased expansion, often starting with a pilot project, of an innovation (e.g., a lifestyle-based diabetes program) into more care settings [2-4]. Canada's International Development Research Centre (IDRC) has suggested the notion of "scaling science" as a complementary domain to implementation science or KT science that is specifically concerned with the optimization of the magnitude, variety, equity, and sustainability of health and social impacts [5-7]. In this review, we define "scaling" as inclusive of all variants of spread, scale or scaling up, scaling out or scaling deep, horizontal, and vertical. By adopting a broad view, we aim to cast a wide net and thereby increase the learning potential of the study.

A variety of scaling models have been developed in the health and social care setting in the last 10 years. However, there is a persistent failure to scale innovations across any health care system, whether in high-, middle-, or low-income countries [5,8]. This failure may be partly due to a lack of scientific knowledge about scaling $[5,9,10]$. An intervention that is proven effective at one scale may have quantitatively or qualitatively new or different impacts at another scale. Research to help predict intervention impacts at scale and to guide the development and execution of scaling strategies is required to improve the success of scaling efforts [5]. Results could support policy makers, program managers, and implementers to identify the right evidence to support their decision-making and plans for scaling.

Many of the existing systematic reviews on scaling in health and social care focus on a specific area of care, and so evidence is fragmented [10-15]. An umbrella review synthesizes the findings of literature reviews already available. Scaling science is moving quickly as new evidence emerges, and thus a living review is also appropriate as it updates the evidence on a continuous basis. We therefore aim to undertake a living umbrella review on scaling science in health and social care. Our proposal satisfies the three guiding criteria for systematic living reviews $[16,17]$. Our study will reconcile all sources of evidence, generalize findings from all types of literature review following a systematic approach and provide a single document that summarizes findings. Additionally, it will facilitate access to the literature and help policy makers and program managers make informed decisions about scaling in health and social care. Finally, it will signal important knowledge gaps and help prioritize future research.

\section{Methods}

We adopted the Joanna Briggs Institute (JBI) methodology for umbrella reviews [18], and we used the Preferred Reporting Items for Systematic Reviews and Meta-Analyses-Protocol (PRISMA-P) guidelines to structure this protocol (Additional file 1) [19]. The study protocol was registered with the International Prospective Register of Systematic Reviews (PROSPERO, CRD42020183774).

\section{Research question}

What is the evidence about scaling in health and social care and what are the knowledge gaps in the literature?

\section{Eligibility criteria}

We will address all types of evidence matching the "PICO" criteria (Participants, Intervention, Comparator, Outcome) and "PICo" (Population, phenomena of Interest and Context) to capture the evidence from quantitative and qualitative reviews.

\section{Participants or population}

All reviews that include primary studies focusing on individuals (e.g., patients or caregivers, health care providers), or health systems, services, or organizations that have been exposed to the scaling of a health or social care innovation.

There will be no restrictions based on sociodemographic factors (e.g., age, ethnicity, socio-economic status) or general health conditions (e.g., comorbidities).

\section{Interventions/phenomena of interest}

No restrictions. We will consider all types of scaling, vertical and horizontal. This umbrella review will not only explore the scaling of health and social care innovations but also any aspect of or any topic relating to such scaling, including (but not limited to) concepts, models, analytical models, tools, cost or impact assessments, and user engagement.

\section{Comparator}

No restrictions. 


\section{Outcomes}

We will consider all outcomes reported in the included reviews (i.e., no restrictions), including health outcomes. We will seek outcomes such as (but not limited to) patients/caregivers and/or health care providers and/or policy makers' perceptions and experiences of barriers, facilitators, acceptability and feasibility of scaling innovations, impact (e.g., adaptability, efficacy, effectiveness), coverage (e.g., proportion of the target population that is reached by the scaling, adoption, fidelity, penetration, or maintenance of the innovation), health outcomes (e.g., impact on morbidity, mortality), patient reported outcomes (e.g., quality of life, satisfaction), and health care resources (e.g., cost-effectiveness of the scaled innovation, cost of staff resources).

\section{Types of study}

We will consider all types of review (quantitative, qualitative, and mixed-methods) that address, synthesize, and summarize evidence in the field of scaling. We define a review as a knowledge synthesis of evidence that includes a clear research question, describes the methods used (which are reproducible) to identify and select the primary research studies, and synthesizes data from its included studies [20, 21]. Reviews can include studies with any research design. We will include all types of review that have been rigorously conducted according to their chosen methodological approach. We will exclude reviews that do not describe their search strategy and inclusion criteria explicitly at the stage of full-text screening. We will also exclude primary research studies, conference abstracts, comments, opinions, letters, and editorials.

\section{Context/setting}

We will include reviews regarding any type of health and social care setting (e.g., home care, community, hospital, primary care, specialized care) in any geographical setting (e.g., rural or urban regions, low-, middle- or highincome countries).

We define "health and social care services" as follows:

Health care consists of services provided in institutional or community settings, any form of access to a health-related service (such as dental, podiatry, or optical services), and access to health care practitioners (such as nurses, physiotherapists, or general practitioners) [22].

Social care consists of interventions that support frail or vulnerable individuals by meeting needs or enabling them to meet needs that arise as a result of physical, mental, or emotional impairment [23].

\section{Search strategy}

Our information specialist (NR) will develop an OvidMEDLINE and Web of Science strategy with input from the project team and a second information specialist. An iterative revision process will be conducted by the members of the research team. Research keywords will include the following: "scaling," "reviews," and "health and social care". We will use the search strategy for "scaling up" previously developed by members of our team. The Canadian Agency for Drugs and Technologies in Health (CADTH) search filter for Systematic Reviews/MetaAnalysis/Health Technology Assessment was adapted for this project [24]. A second information specialist will review the search strategy using the Peer Review of Electronic Search Strategies (PRESS) tool [25]. Comments will be integrated in a final version of the search strategy. The final version will be approved by the team members. Once approved, this search strategy will be translated into the other databases mentioned below. The exact search terms will be recorded as the search strategy is refined. This protocol only includes the search strategy conducted in one database (Additional file 2). A further systematic literature search will be performed to identify published studies in the following electronic bibliographic databases: MEDLINE (Ovid), Embase, PsychINFO (Ovid), CINAHL (EBSCO), Web of Science, The Cochrane Library, Sociological Abstract (Proquest), Academic Search Premier (EBSCO), and Proquest Dissertations \& Theses Global. No language restriction will be applied. We will search from inception onwards.

Additionally, to identify the grey literature, we will search the websites of relevant organizations such as the World Health Organization (WHO), Global Reporting Initiative, the UK's National Institute for Care and Excellence (NICE), Australia NSW Government, the Institut National d'Excellence en Santé et Services Sociaux (INESSS) in Quebec, Canada, the Canadian Foundation for Healthcare Improvement (CFHI), Canada's IDRC, the CADTH's Grey Matters checklist, and clinical trial registries. We will contact experts in the field by email for additional data. In addition to searching databases, the reference list of each included review will be reviewed.

\section{Selection process}

The search results will be imported and stored in an EndNote X9 library for reference management and duplicate removal $[26,27]$. The resulting records will be exported to the Internet-based system Covidence for the selection process [28]. The team will develop a selection grid. It will be adjusted, if necessary, before the screening of titles and abstracts of all articles. All selection criteria will be discussed by reviewers to ensure common understanding. A pilot screening (titles and abstracts) of $2.5 \%$ random sample reviews will be completed. 
Discrepancies will be resolved by consensus or by a third reviewer if necessary.

First, two independent reviewers will screen titles and abstracts of identified reviews against the eligibility criteria. Articles with abstracts that do not appear to meet the criteria for exclusion, or are ambiguous, or have missing abstracts, will be retained and reviewed in full. Second, full-text examination of the remaining reviews will be assessed for eligibility. The reviewers' full understanding of the selection criteria will be validated again before beginning this stage. In case of an "unclear" response regarding the eligibility of studies, authors will resolve disagreements through discussion and, if necessary, consult a third senior author. Any reasons for exclusion will be recorded in Excel.

Articles that are not available electronically will be ordered via interlibrary loan. We will contact the corresponding author if an email is available.

\section{Data extraction}

Once the reviews have been selected for inclusion, two reviewers will independently conduct the data extraction. We will develop a data extraction form based on the JBI form for review of systematic reviews. A standardized, pre-piloted form will be used to extract data from the included reviews for assessment of study quality and evidence synthesis. A pilot data extraction will be conducted on $5 \%$ of selected reviews until conclusive results are agreed on between reviewers. Any disagreement between the reviewers will be resolved through discussion; if consensus is not reached, they will consult a third senior reviewer. We will not extract data from the primary studies included in the reviews. We will summarize the review findings but we will not resynthesize the results of primary studies. Extracted information will include:

- Review characteristics: author, year of publication, country, type of review; aim of the study; language; PICO or PICo; number and characteristics of participants (e.g. sexas a biological variable, age); setting and context; number and type of databases sourced and searched; date range of database searching; citation index; number and study design of primary studies included in each review; instrument used to appraise the primary studies and the rating of their quality; method of synthesis/ analysis used to synthesize the evidence; synthesis/ summary of results; heterogeneity if applicable; mention by the authors of research needs or gaps in the review.

- Focus of the reviews: infrastructure (e.g., policies, guidelines, human and material resources); concepts and models (e.g., definition, conceptual model, framework); measurement (e.g., tools, scalability, cost); analytical methods (e.g., mathematical approach); descriptive (e.g., perceptions, barriers, facilitators, acceptability); patient engagement (e.g., authorship, study's reporting on patient advocates) and scaled interventions (e.g., strategies, training, process, coverage, feasibility, effectiveness).

\section{Assessing quality of reviews}

Two independent reviewers will use AMSTAR2 [29] to assess the methodological quality of included reviews and ROBIS [30] for the risk of bias. Based on the nature of the included reviews, we will explore the relevance of using the GRADE assessment system [31, 32]. Disagreements between the reviewers over the quality or the risk of bias in particular studies will be resolved by discussion, with involvement of a third author where necessary. AMSTAR2 is a validated, widely used 16item instrument for assessing reviews of reviews. ROBIS contains 24 questions divided into three phases. Phase 1 assesses relevance (optional) and verifies if the research question matches the umbrella review's PICO; phase 2 identifies concerns with the review process; and phase 3 judges risk of bias in the review. Phase 2 and 3 questions are answered with the following options: yes, probably yes, probably no, no, no information. The concerns regarding phase 2 domains and phase 3 are classified as high, low, or unclear.

\section{Data synthesis}

We will use the Preferred Reporting Items for Systematic Reviews and Meta-Analyses (PRISMA) flow diagram to describe the process of study selection [33]. We will use tables to describe characteristics of included studies (e.g., first author's name, year of publication, study aim) and analysis details (e.g., appraisal instrument used, methods of analysis, results summary, authors comments, heterogeneity of the results), and we will synthesize the data narratively using categories of scaling evidence that have emerged from the included reviews (e.g., descriptive reviews of scaling, reviews of barriers and facilitators, reviews of determinants, reviews of concepts, reviews of frameworks, reviews of economic evaluation, reviews of scaling interventions, reviews of scaling measures, reviews of effects of scaling interventions, reviews of scalability).

A meta-analysis will not be performed, given that pooling the results of qualitative and quantitative reviews can introduce significant overlap and bias. We will clearly indicate the overlap between primary studies within the reviews in the tables, and we will develop a citation matrix. Because our purpose is to identify evidence about scaling in health and social care in the literature, we will present the whole body of knowledge 
and include the results of all the reviews, regardless of the overlap across primary studies [34].

\section{Living update}

Based on the series Living Systematic Reviews [17, 35-38], the search for new reviews will be repeated at regular intervals by the coordinator team, i.e., tri-monthly searches of bibliographic databases and every six months for the grey literature. The studies identified during these updated searches will be analyzed using the same eligibility criteria as used in the initial search. The new information will be integrated at least once a year, when it will be combined with all the "stand by" information. If the information has an effect on the evidence, it will be included in the review and an update will be published.

\section{Discussion}

This review will help to build the science of scaling in health and social care. We are not aware of any other umbrella or systematic review addressing this issue. As an international group of researchers, policy-makers, and funders, we are aware of the increasing importance of the science of scaling and its potential for optimizing the benefits of health research for individuals, institutions, and systems. Anticipated challenges for this review include the complexity of summarizing the research syntheses from such a diversity of review types and topics and to determine the point when newly identified elements of scaling science can be described as common themes. Important protocol amendments will be documented and noted in the discussion. The dissemination plan is to present the results through publications in peerreview journals, relevant networks and social media, and presentations at national and international conferences.

\footnotetext{
Abbreviations

JBI: Joanna Briggs Institute; AMSTAR2: A Measurement Tool to Assess Systematic Reviews 2; ROBIS: Risk of Bias in Systematic Reviews; PROSPERO: International Prospective Register of Systematic Reviews; KT: Knowledge Translation; IDRC: International Development Research Centre; GRADE: Grading of Recommendations, Assessment, Development and Evaluation; PRISMA: Preferred Reporting Items for Systematic Reviews and Meta-Analyses; PRISMA-P: Preferred Reporting Items for Systematic Reviews and Meta-Analyses-Protocol; PICO: Participants, Intervention, Comparator, Outcome; PICo: Population, phenomena of Interest and Context; PRESS: Peer Review of Electronic Search Strategies; WHO: World Health Organization; NICE: National Institute for Care and Excellence (UK); INESSS: Institut National d'Excellence en Santé et Services Sociaux (Canada); CFHI: Canadian Foundation for Healthcare Improvement; NSW: New South Wales; CADT $\mathrm{H}$ : Canadian Agency for Drugs and Technologies in Health; MLS: Masters in Library Science; CIHR: Canadian Institutes of Health Research; FRQ-S: Fonds de recherche du Québec-Santé
}

\section{Supplementary Information}

The online version contains supplementary material available at https://doi. org/10.1186/s13643-021-01813-3.

Additional file 1. PRISMA-P (Preferred Reporting Items for Systematic review and Meta-Analysis Protocols) 2015 checklist: recommended items to address in a systematic review protocol*.
Additional file 2. Search strategy.

Acknowledgements

We thank Frederic Bergeron, MLS, for the PRESS evaluation and the peer review and for performing the search strategy. We also thank Louisa Blair for her kind editorial help with the manuscript.

\section{Authors' contributions}

Members of the Executive Committee FL, ABC, AG, and HTVZ contributed to the conception and design of the study. FL, KVP, and FB drafted the protocol. All authors critically reviewed the original protocol and subsequent versions. All authors read and approved the final manuscript.

\section{Funding}

This research project is funded by the Canadian Institutes of Health Research (CIHR) Foundation Grant (\#FDN-159937). ABC is funded by the Fonds de recherche du Québec-Santé (FRQ-S). AG is funded by the CIHR PatientOriented Research Awards. FL holds a Tier 1 Canada Research Chair in Shared Decision Making and Knowledge Translation. The financial providers are not involved in the project.

Availability of data and materials

The datasets used and/or analyzed during the current study are available from the corresponding author on reasonable request.

\section{Declarations}

Ethics approval and consent to participate

Not applicable.

\section{Consent for publication}

Not applicable. The manuscript does not contain any individual person's data.

\section{Competing interests}

The authors declare that they have no competing interests.

\section{Author details}

${ }^{1}$ VITAM - Centre de recherche en santé durable, Université Laval, Pavillon Landry-Poulin - 2525, Chemin de la Canardière, Quebec, QC, Canada. ${ }^{2}$ Tier 1 Canada Research Chair in Shared Decision Making and Knowledge Translation, Université Laval, Quebec, QC, Canada. ${ }^{3}$ Health and Social Services Systems, Knowledge Translation and Implementation component of the Quebec SPOR-SUPPORT Unit, Université Laval, Quebec, QC, Canada. ${ }^{4}$ Department of Family Medicine and Emergency Medicine, Université Laval, Quebec, QC, Canada. ${ }^{5}$ International Development Research Centre, Ottawa, ON, Canada. ${ }^{6}$ Faculty of Medicine and Health Sciences, Stellenbosch University, Tygerberg, South Africa. ${ }^{7}$ School of Public Health, University of Sydney, Sydney, NSW, Australia. ${ }^{8}$ Centre for Epidemiology and Evidence, NSW Ministry of Health, Sydney, NSW, Australia. ${ }^{9}$ School of Medicine and Public Health, University of Newcastle, Callaghan, NSW, Australia. ${ }^{10}$ Hunter Medical Research Institute, New Lambton Heights, NSW, Australia. ${ }^{11}$ Hunter New England Population Health, Wallsend, NSW, Australia.

Received: 2 March 2021 Accepted: 10 September 2021

Published online: 30 September 2021

\section{References}

1. Greenhalgh T, Papoutsi C. Spreading and scaling up innovation and improvement. BMJ. 2019;365:12068. https://doi.org/10.1136/bmj.I2068.

2. WHO. Nine steps for developing a scaling-up strategy. ExpandNet. 2010.

3. Barker PM, Reid A, Schall MW. A framework for scaling up health interventions: lessons from large-scale improvement initiatives in Africa. Implement Sci. 2016;11(1):12. https://doi.org/10.1186/s13012-016-0374-x.

4. Milat AJ, Newson R, King L, Rissel C, Wolfenden L, Bauman A, et al. A guide to scaling up population health interventions. Public Health Res Pract. 2016; 26(1):e2611604. https://doi.org/10.17061/phrp2611604.

5. McLean R, Gargani J. Scaling impact: innovation for the public good. 1st ed. London; New York: Routledge; 2019. p. 256. https://doi.org/10.4324/978042 9468025. 
6. McLean R, Gargani J. In Brief: Scaling Science. Ottawa: International Development Research Centre; 2020. http://hdl.handle.net/10625/59067. Accessed 11 Feb 2021.

7. Gogovor A, Zomahoun HTV, Ben Charif A, McLean RKD, Moher D, Milat A et al. Essential items for reporting of scaling studies of health interventions (SUCCEED): protocol for a systematic review and Delphi process. Syst Rev. 2020;9(1):11. https://doi.org/10.1186/s13643-019-1258-3.

8. Milat AJ, Bauman A, Redman S. Narrative review of models and success factors for scaling up public health interventions. Implement Sci. 2015;10(1): 113. https://doi.org/10.1186/s13012-015-0301-6.

9. Milat AJ, King L, Bauman A, Redman S. Scaling up health promotion interventions: an emerging concept in implementation science. Health Promot J Austr. 2011;22(3):238. https://doi.org/10.1071/HE11238.

10. Ben Charif A, Zomahoun HTV, LeBlanc A, Langlois L, Wolfenden L, Yoong $S L$, et al. Effective strategies for scaling up evidence-based practices in primary care: a systematic review. Implement Sci. 2017;12(1):139. https://doi. org/10.1186/s13012-017-0672-y.

11. Zakiyah N, van Asselt AD, Roijmans F, Postma MJ. Economic evaluation of family planning interventions in low and middle income countries; a systematic review. PLoS One. 2016;11(12):e0168447. https://doi.org/10.1371/ journal.pone.0168447.

12. McCrabb S, Lane C, Hall A, Milat A, Bauman A, Sutherland R, et al. Scalingup evidence-based obesity interventions: a systematic review assessing intervention adaptations and effectiveness and quantifying the scale-up penalty. Obes Rev. 2019;20(7):964-82. https://doi.org/10.1111/obr.12845.

13. Hempel S, O'Hanlon C, Lim YW, Danz M, Larkin J, Rubenstein L. Spread tools: a systematic review of components, uptake, and effectiveness of quality improvement toolkits. Implement Sci. 2019;14(1):83. https://doi.org/1 0.1186/s13012-019-0929-8.

14. Bulthuis SE, Kok MC, Raven J, Dieleman MA. Factors influencing the scale-up of public health interventions in low- and middle-income countries: a qualitative systematic literature review. Health Policy Plan. 2020;35(2):21934. https://doi.org/10.1093/heapol/czz140.

15. Carroll G, Safon C, Buccini G, Vilar-Compte M, Teruel G, Perez-Escamilla R. A systematic review of costing studies for implementing and scaling-up breastfeeding interventions: what do we know and what are the gaps? Health Policy Plan. 2020;35(4):461-501. https://doi.org/10.1093/heapol/czaa005.

16. Elliott JH, Turner T, Clavisi O, Thomas J, Higgins JP, Mavergames C, et al. Living systematic reviews: an emerging opportunity to narrow the evidence-practice gap. PLoS Med. 2014;11(2):e1001603. https://doi.org/10.13 71/journal.pmed.1001603.

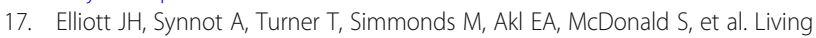
systematic review: 1. Introduction-the why, what, when, and how. J Clin Epidemiol. 2017;91:23-30. https://doi.org/10.1016/j.jclinepi.2017.08.010.

18. Aromataris E, Fernandez R, Godfrey C, Holly C, Khalil H. P. T. Chapter 10: Umbrella reviews. In: Aromataris E, Munn Z, editors. Joanna Briggs Institute Manual for Evidence Synthesis; 2020. Available from: https:/synthesismanual.jbi.global.

19. Moher D, Shamseer L, Clarke M, Ghersi D, Liberati A, Petticrew M, et al. Preferred reporting items for systematic review and meta-analysis protocols (PRISMA-P) 2015 statement. Syst Rev. 2015;4(1):1. https://doi.org/10.1186/204 6-4053-4-1.

20. Grant MJ, Booth A. A typology of reviews: an analysis of 14 review types and associated methodologies. Health Info Libr J. 2009;26(2):91-108. https:// doi.org/10.1111/j.1471-1842.2009.00848.x.

21. Sutton A, Clowes M, Preston L, Booth A. Meeting the review family: exploring review types and associated information retrieval requirements. Health Info Libr J. 2019;36(3):202-22. https://doi.org/10.1111/hir.12276.

22. Evans $D$. The provision of health and social care services for older people by respite providers. Contemp Nurse. 2013;45(2):255-63. https://doi.org/10.51 72/conu.2013.45.2.255

23. Burge P, Netten A, Gallo F. Estimating the value of social care. J Health Econ. 2010;29(6):883-94. https://doi.org/10.1016/j.jhealeco.2010.08.006.

24. Canadian Agency for Drugs and Technologies in Health. Strings Attached: CADTH's Database Search Filter. CADTH Evidence Driven. 2020.

25. McGowan J, Sampson M, Salzwedel DM, Cogo E, Foerster V, Lefebvre C. PRESS Peer Review of Electronic Search Strategies: 2015 Guideline Statement. J Clin Epidemiol. 2016;75:40-6. https://doi.org/10.1016/j.jclinepi.2 016.01.021.

26. Bramer WM, Giustini D, de Jonge GB, Holland L, Bekhuis T. De-duplication of database search results for systematic reviews in EndNote. J Med Libr Assoc. 2016;104(3):240-3. https://doi.org/10.3163/1536-5050.104.3.014.
27. EndNote, version X9. Clarivate analytics. 2018.

28. Covidence systematic review software. Melbourne: Veritas Health Innovation. Available from: www.covidence.org. Accessed 11 Feb 2021.

29. Shea BJ, Reeves BC, Wells G, Thuku M, Hamel C, Moran J, et al. AMSTAR 2: a critical appraisal tool for systematic reviews that include randomised or non-randomised studies of healthcare interventions, or both. BMJ. 2017;358: j4008.

30. Whiting P, Savovic J, Higgins JP, Caldwell DM, Reeves BC, Shea B, et al. ROBIS: a new tool to assess risk of bias in systematic reviews was developed. J Clin Epidemiol. 2016;69:225-34. https://doi.org/10.1016/j. jclinepi.2015.06.005.

31. Guyatt GH, Oxman AD, Vist GE, Kunz R, Falck-Ytter $Y$, Alonso-Coello P, et al. GRADE: an emerging consensus on rating quality of evidence and strength of recommendations. BMJ. 2008;336(7650):924-6. https://doi.org/10.1136/ bmi.39489.470347.AD.

32. Andrews JC, Schunemann HJ, Oxman AD, Pottie K, Meerpohl JJ, Coello PA, et al. GRADE guidelines: 15. Going from evidence to recommendationdeterminants of a recommendation's direction and strength. J Clin Epidemiol. 2013;66(7):726-35. https://doi.org/10.1016/j.jclinepi.2013.02.003.

33. Moher D, Liberati A, Tetzlaff J, Altman DG, Group P. Preferred reporting items for systematic reviews and meta-analyses: the PRISMA statement. J Clin Epidemiol. 2009;62(10):1006-12. https://doi.org/10.1016/j.jclinepi.2009. 06.005 .

34. Pollock M, Fernandes R, Becker L, Pieper D, Hartling L. Chapter V: Overviews of reviews. In: Higgins J, Thomas J, Chandler J, Cumpston M, Li T, Page M, et al., editors. Cochrane Handbook for Systematic Reviews of Interventions version 60 (updated March 2020); 2020. Available from: www.training.cochra ne.org/handbook

35. Thomas J, Noel-Storr A, Marshall I, Wallace B, McDonald S, Mavergames C, et al. Living systematic reviews: 2. Combining human and machine effort. J Clin Epidemiol. 2017;91:31-7. https://doi.org/10.1016/j.jclinepi.2017.08.011.

36. Simmonds M, Salanti G, McKenzie J, Elliott J, Living Systematic Review N. Living systematic reviews: 3. Statistical methods for updating meta-analyses. J Clin Epidemiol. 2017;91:38-46. https://doi.org/10.1016/j.jclinepi.2017.08.008.

37. Akl EA, Meerpohl JJ, Elliott J, Kahale LA, Schunemann HJ, Living systematic review N. Living systematic reviews: 4. Living guideline recommendations. J Clin Epidemiol. 2017:91:47-53. https://doi.org/10.1016/j.jclinepi.2017.08.009.

38. Millard T, Synnot A, Elliott J, Green S, McDonald S, Turner T. Feasibility and acceptability of living systematic reviews: results from a mixed-methods evaluation. Syst Rev. 2019;8(1):325. https://doi.org/10.1186/s13643-019-12485.

\section{Publisher's Note}

Springer Nature remains neutral with regard to jurisdictional claims in published maps and institutional affiliations.

Ready to submit your research? Choose BMC and benefit from:

- fast, convenient online submission

- thorough peer review by experienced researchers in your field

- rapid publication on acceptance

- support for research data, including large and complex data types

- gold Open Access which fosters wider collaboration and increased citations

- maximum visibility for your research: over $100 \mathrm{M}$ website views per year

At BMC, research is always in progress.

Learn more biomedcentral.com/submission 